\title{
New Patchy Particle Model with Anisotropic Patches for Molecular Dynamics Simulations: Application to a Coarse-Grained Model of Cellulose Nanocrystal
}

Nicolas Rolland, Alexandar Mehandzhiyski, Mohit Garg, Mathieu Linares and Igor Zozoulenko

The self-archived postprint version of this journal article is available at Linköping University Institutional Repository (DiVA):

http://urn.kb.se/resolve?urn=urn:nbn:se:liu:diva-167676

N.B.: When citing this work, cite the original publication.

Rolland, N., Mehandzhiyski, A., Garg, M., Linares, M., Zozoulenko, I., (2020), New Patchy Particle Model with Anisotropic Patches for Molecular Dynamics Simulations: Application to a Coarse-Grained Model of Cellulose Nanocrystal, Journal of Chemical Theory and Computation, 16(6), 3699-3711. https://doi.org/10.1021/acs.jctc.oc00259

Original publication available at:

https://doi.org/10.1021/acs.jctc.oco0259

Copyright: American Chemical Society http://pubs.acs.org/ 


\title{
New Patchy Particle Model with Anisotropic
}

\section{Patches for Molecular Dynamics Simulations:}

\section{Application to a Coarse-Grained model of Cellulose Nanocrystal}

Nicolas Rolland, ${ }^{*, \dagger}$ Aleksandar Y. Mehandzhiyski, ${ }^{\dagger}$ Mohit Garg, ${ }^{\dagger}$ Mathieu Linares, $^{\dagger,+, \uparrow}$ and Igor $\vee$. Zozoulenko*,,$\S$

$\dagger$ Laboratory of Organic Electronics, ITN, Linköping University, SE-601 74 Norrköping, Sweden

$\ddagger$ Scientific Visualization Group, ITN, Linköping University, SE-601 74 Norrköping, Sweden

【Swedish e-Science Research Centre (SeRC), Linköping University, SE-581 83 Linköping, Sweden

$\S$ Wallenberg Wood Science Center, Linköping University, SE-601 74 Norrköping, Sweden

E-mail: nicolas.rolland@liu.se; igor.zozoulenko@liu.se

\begin{abstract}
Self-assembly is ubiquitous in nature and underlies the formation of many complex systems from the molecular to the macroscopic scale. Kern-Frenkel like patchy particles are powerful models to investigate this phenomenon by computational methods such as Monte-Carlo or Molecular Dynamics simulations. However, in these models the interactions are mediated by circular patches at the particles surface, which can be hardly mapped to realistic systems, containing for instance faceted particles with rectangular
\end{abstract}


surfaces. In this paper we extend the model to take into account such geometries, and we use it to build a supra Coarse-Grained model of Cellulose Nanocrystal where the interactions are parametrized against All-Atomistic Molecular Dynamics simulations. The formation of cholesteric ribbons and defects in cholesteric droplets of Cellulose Nanocrystal are investigated and confirm experimental behavior reported in the literature. The flexibility of this new patchy particle model makes it a powerful tool to develop supra Coarse-Grained models of self-assembly for large space and time scales and should find a broad range of applications for self-assembly in chemical and biological systems.

\section{Introduction}

Self-assembly, which refers to the autonomous organization of building blocks into specific patterns, is ubiquitous in nature and underlies the formation of many complex biological systems. ${ }^{1}$ It can provide an efficient way of manufacturing complex objects at all scales and constitutes a totally new paradigm into technologies. ${ }^{2}$ Synthesis relying on such selfassemblies has already been applied successfully on a very broad range of length scales, and at the micrometric scale for instance, many efforts have been devoted to develop so-called patchy particles as building blocks. ${ }^{3-6}$ They are particles, micrometric colloids essentially, with anisotropic shapes or surface functionalizations, which can self-assemble into a vast zoology of structures, such as $2 \mathrm{D}$ crystals, ${ }^{7-11} 3 \mathrm{D}$ crystals, ${ }^{12-16}$ or helices ${ }^{17-21}$ due to the anisotropic interactions between particles. They can be described theoretically by the KernFrenkel patchy particle model, ${ }^{22}$ and in particular the tetravalent Kern-Frenkel model has been widely used to investigate the phase diagram of tetrahedral liquids (e.g. water or silicon). ${ }^{23-29}$ This model, illustrated in Figure 1(a), is a combination of the hard sphere model (blue particles) with four attractive square well patches (pinkish cones). A patch $k$ on a particle $i$ can interact with another patch $l$ on a different particle $j$ providing that they are facing each other, and thus promoting a certain relative orientation of two adjacent 
particles, leading for instance to the formation of a diamond structure. More precisely, the Kern-Frenkel interaction potential $U_{K F}$ between particle $i$ and $j$ is the sum of all patches interactions:

$$
U_{K F}=\sum_{k=1}^{4} \sum_{l=1}^{4} U_{k l}
$$

where the patch-patch interaction potential reads

$$
\begin{aligned}
& U_{k l}= \\
& \begin{cases}\infty & \text { if }\left|\overrightarrow{r_{i j}}\right| \leq \sigma \\
-\epsilon & \text { if } \sigma<\left|\overrightarrow{r_{i j}}\right| \leq \sigma+\delta \text { and } \theta_{i, k} \leq \Omega \text { and } \theta_{j, k} \leq \Omega \\
0 & \text { if }\left|\overrightarrow{r_{i j}}\right|>\sigma+\delta\end{cases}
\end{aligned}
$$

with

$$
\begin{aligned}
& \cos \left(\theta_{i, k}\right)=\frac{-\overrightarrow{r_{i j}} \cdot \hat{p_{i, k}}}{\left|\overrightarrow{r_{i j}}\right|} \\
& \cos \left(\theta_{j, l}\right)=\frac{\overrightarrow{r_{i j}} \cdot \hat{p_{j, l}}}{\left|\overrightarrow{r_{i j}}\right|}
\end{aligned}
$$

Patchy particles can be used fundamentally as a mesoscale model for atoms (so-called colloidal atoms ${ }^{6}$ ), but they also have practical applications linked to their properties, optics or photonics for instance. ${ }^{30-32}$ Another class of self-assembled materials that has been recognized instrumental in the technology of optics are the liquid crystals. ${ }^{33}$ Here, there is a molecular self-assembly of molecules in solution, the mesogens, into a mesophase possessing both the properties of a liquid and of a crystalline solid. ${ }^{34}$ Among these mesophases, 

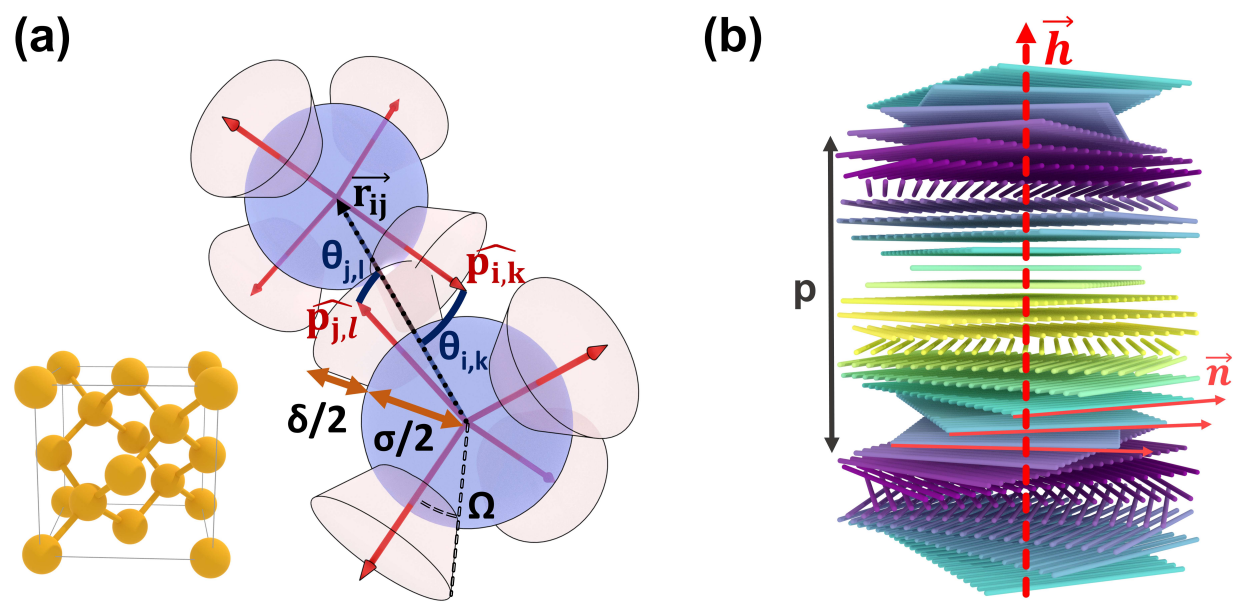

Figure 1: (a) Kern-Frenkel patchy particle model for tetravalent particles. In certain condition these particles can self-assemble into a diamond structure as depicted at the bottom left. (b) Schematic of a cholesteric liquid crystal of rod-like molecules.

cholesteric liquid crystals of rod-like molecules, discovered in the late 19th by Reinitzer, appear as a fascinating material with great potential applications. ${ }^{35}$ This phase, illustrated in Figure 1(b), can be seen as a stack of horizontal layers along a vertical axis $\vec{h}$. All rods in a layer have the same orientation, denoted by the director field $\vec{n}$, and from one layer to the other $\vec{n}$ slightly rotates around the axis $\vec{h}$ to form a helical structure. The length $p$ along the axis $h$ such that the director has rotated $360^{\circ}$, the so-called cholesteric pitch, as well as the sense of rotation of the director, right-handed or left-handed (right-handed in Figure 1(b)), are the principal factors that will affect the general optical properties of the material: Bragg diffraction due to the periodical structure and induced circular dichroism. Cellulose has emerged as one of the most promising materials to fabricate such system due to its low-cost, abundance in nature, biodegradability, renewability, recyclability and already well-established industrial use. ${ }^{36}$ In order to obtain the cholesteric behavior of cellulose, one can prepare an aqueous suspension of Cellulose Nanocrystal (CNC) by acid hydrolysis of cellulose fibers extracted from wood, plants or bacteria followed by a mechanical stirring. ${ }^{37}$ Subsequent solvent evaporation will lead to the formation of a $\mathrm{CNC}$ cholesteric phase that will be retain in the final dry state. Despite the apparent simplicity of the process, there are still a lot of unresolved questions regarding the phase transformation thermodynamics 
and kinetics, and how the final morphology of the cholesteric phase relates to the system parameters: CNC morphology, lengths distribution and surface charges (induced by the acid hydrolysis), and ionic strength of the solvent. ${ }^{38}$ As a result, it remains quite challenging to obtain reproducible pitch and films with uniform optical properties. Yet, a lot of studies have been concerned with the molecular modeling of CNC by mean of All-Atomistic (AA) Molecular Dynamics (MD) simulations ${ }^{39-48}$ or Coarse-Grained (CG) MD, ${ }^{49-56}$ providing essential insights into the properties of an individual CNC as well as the interactions within a small bundle of $\mathrm{CNC}$. However, the computational cost required to investigate the large scale ordering during formation of the cholesteric phase has remained a barrier to model this phase transformation. Only recently we had developed a supra-CG molecular model of CNC able to simulate hundreds of CNC, reaching the micro scale both in space and time, although the self-assembly into the cholesteric phase itself remains challenging. ${ }^{57}$

At a considerably longer length scale, a significant effort has been made for some 70 years, starting with the seminal work of Onsager, to build a theoretical understanding of the cholesteric ordering in liquid crystals. ${ }^{58-71}$ These models, essentially built on hard particles representations, are able to explain the formation of cholesteric liquid crystals but they are then missing a link with the microscopic molecular structure and they are unable to take into account fine details such as molecules flexibility or solvent ionic strength. More recently, CG-MD simulations of model systems such as helical Yukawa rods ${ }^{72,73}$ or flexible chains with helical interactions ${ }^{74,75}$ have appeared as an interesting compromise between the aforementioned models and molecular CG models like existing for the CNC. However, in these models the introduction of the molecule chirality is done by considering only one helical arrangement of spherical beads around the molecule core, which still can be hardly mapped to any real molecular structure. On the other hand, Kern-Frenkel-like patchy particles models for MD have been successfully developed and appear to be very powerful and versatile models to handle complex self-assembly process, being the formation of helical structures, hexagonal columnar and body-centered tetragonal structures, graphene-like two-dimensional structures, 
diamond lattices, urchin-like cluster structures, ordered hamburger-like structures and linear chain-like structures. ${ }^{76,77}$

In this work, we develop a new Kern-Frenkel-like patchy particle model for MD and apply it to the CG modeling of CNC. We start by introducing the CG beads definition based a molecular description of the $\mathrm{CNC}$. We then present the interaction potentials that we have used and the parametrization of the force-field against AA-MD simulations following the methodology that we have used previously to build our supra-CG model of CNC. ${ }^{57}$ Finally, we illustrate the potential of the new model to investigate cholesteric ordering in $\mathrm{CNC}$, by simulating 1D cholesteric ribbons and a 3D cholesteric nanodroplet.

\section{Supra Coarse-Grained model}

The essence of the supra CG model is summarized by the central part of Figure 2. A CNC is a long fiber-like twisted molecule, that we cut into small sections represented by the greyish nearly hexagonal prisms. These sections are modeled by ellipsoidal CG beads (brownish particles). The beads are decorated with six patches (forming a ball around each bead) that introduce attractive interactions between the various CNC surfaces (different patch colors mean different types of surfaces), and lead to the self-assembly of the CNC. We shall now present the beads definition in details and the interaction potentials that we use.

\subsection{Beads definition}

A CNC comprises a self-assembly of many polysaccharide chains, the cellulose polymers, as illustrated in Figure 2. Two allomorphs exist for the native CNC, respectively the cellulose $I_{\beta}$ and $I_{\alpha}$, and so far there is no consensus regarding the cross-sectional morphology of the CNC. In this study, we considered the 36-chains $I_{\beta}$ model of Ding and Himmel ${ }^{78}$ as shown

in Figure 2(a), because: (i) NMR spectroscopy suggests that the $I_{\beta}$ crystalline allomorph is the one encountered in higher plants, ${ }^{79}$ (ii) this shape of the $\mathrm{CNC}$ is a good study-case 
to demonstrate the flexibility of our novel approach since it has a non-regular cross-section (otherwise we could use the "standard" Kern-Frenkel patchy particle model). The $I_{\beta}$ CNC has a monoclinic crystallographic cell, depicted in Figure $2(\mathrm{a})$, with $\mathrm{a}=7.784 \AA$, $\mathrm{b}=8.201$ $\AA, \mathrm{c}=10.38 \AA, \alpha=\beta=90^{\circ}, \gamma=96.5^{\circ},{ }^{80}$ and features three different surfaces, the (100), often referred as hydrophobic, and the (110) and (1-10), often referred as hydrophilic. The length of the CNC along the $c$-axis (in the $x$-direction in Figure 2(a)) can vary from tens of nanometers to several micrometers. ${ }^{81}$ Beads are defined in a very straightforward way by simply cutting the nanocrystal into several sections along the $c$-crystallographic direction. In this way, each bead groups 36 glucose units (in the $a b$ crystallographic plane) $\times 8$ glucose unit (along the $c$-crystallographic direction) and contains around 6000 atoms.

Since the groups of atoms represented by the beads are highly anisotropic and should interact in very different ways depending on their relative orientations, we introduced finite size aspherical particles to track the beads dynamics in the MD simulations. All that is required to describe a finite size aspherical particle is its mass and position, just like in a point-particle MD simulation, but also its orientation and its moment of inertia. Then it can rotate due to the torques coming from interactions with other particles. One could directly calculate the exact moment of inertia of the group of atoms represented by the beads, but for simplicity we simply approximated the bead shape by an ellipsoid as depicted in Figure 2(a). The calculation of the resulting moment of inertia is straightforward and is handled automatically by the LAMMPS software that we used to implement our new supra CG model. ${ }^{82}$ The orientation of the beads is given by the three principal ellipsoid semi-axes $\left(\overrightarrow{e_{x}}\right.$, $\left.\overrightarrow{e_{y}}, \overrightarrow{e_{z}}\right)$, whose lengths are (41.52 $\left.\AA, 45.54 \AA, 27.05 \AA\right)$. It is worth noting that the ellipsoid shape drives the dynamics of the beads through the moment of inertia that will appear in Newton's law of motion, but it does not represent any kind of interaction between the beads. The way two particles interact when their surfaces are close to each other is described by the potentials that will be introduced in the following. Based on this bead definition, the energy function that will drive the MD simulations is written as 


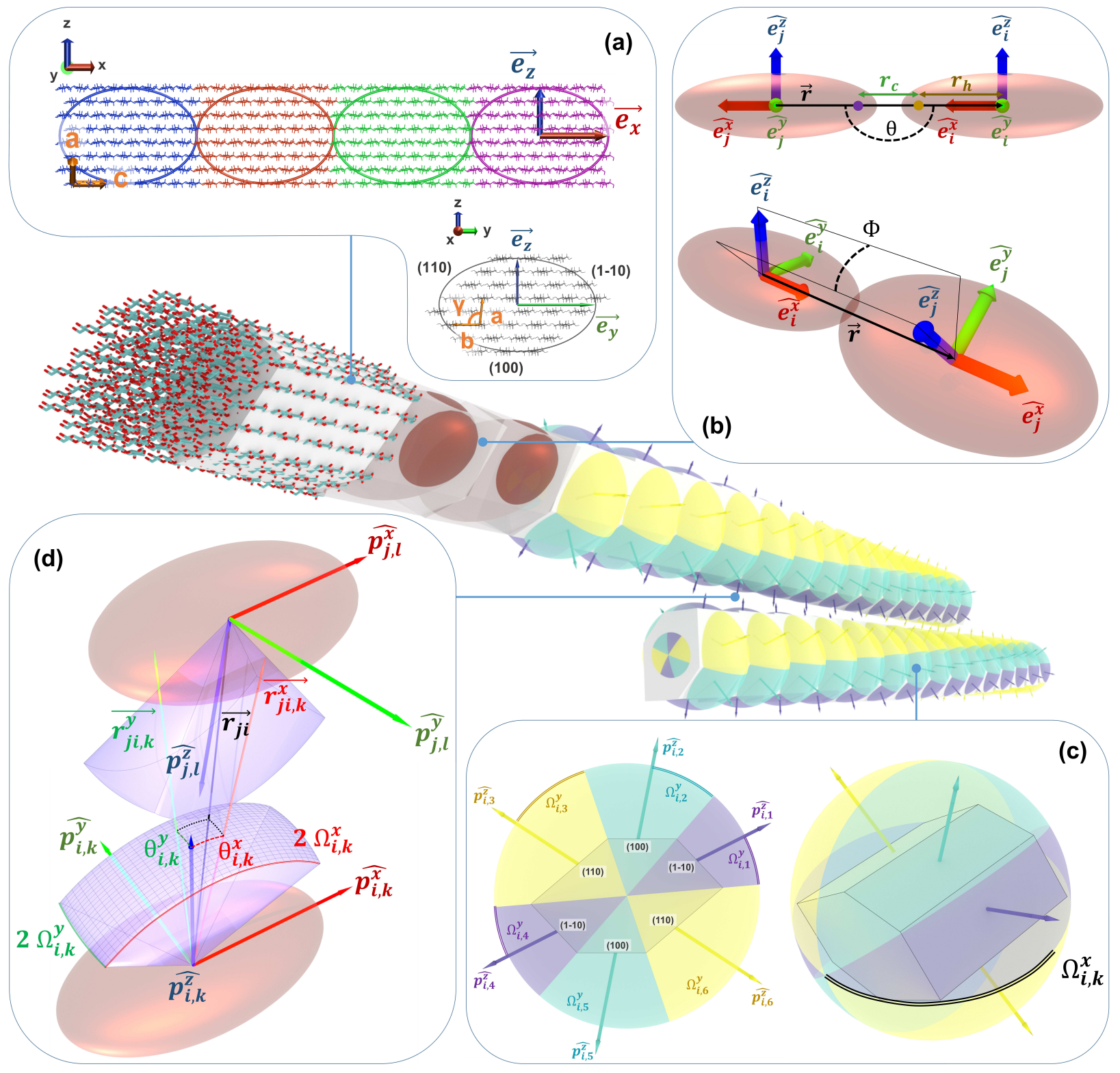

Figure 2: Supra Coarse-Grained patchy particle model of cellulose nanocrystal. The central part shows two interacting CNC. At the forefront, the All-Atomistic representation of the $\mathrm{CNC}$ is shown, where cyan atoms are carbon and red atoms are oxygen (hydrogen atoms are omitted for clarity). (a) Cross-section (top) and side-view (bottom) of a part of the CNC. Colors on the side-view show how the atoms are grouped into CG beads. Ellipsoidal CG beads (brownish particles on the central picture) approximate the nearly hexagonal prismatic shape (greyish particles on the central picture) of the groups of atoms. (b) Geometrical parameters involved in the calculation of the bonded interaction between adjacent CG beads $i$ and $j$ in the same CNC, for the harmonic bond and cosine harmonic angle potentials (top), and for the dihedral potential (bottom). (c) Geometrical definition of the patches decorating the CG beads, seen into cross-section (left) and perspective (right) views. The 6 circular sectors represent various patches, each color coding for a specific patch type / surface type. The purple nearly hexagonal prism highlights the shape of the group of atoms modeled by the CG bead. (d) General representation of the interaction between a patch $k$ on bead $i$ and a patch $l$ on bead $j .\left(p_{i, k}^{\hat{x}}, p_{i, k}^{\hat{y}}, p_{i, k}^{\hat{z}}\right)$ and $\left(p_{j, l,}^{\hat{x}} p_{j, l}^{\hat{y}}, p_{j, l}^{\hat{z}}\right)$ are the patch orientations, and the aperture angles $2 \Omega_{i, k}^{x}$ and $2 \Omega_{i, k}^{y}$ of patch $k$ are highlighted. 


$$
U_{\text {tot }}=\sum_{i=1}^{N_{\text {bond }}} U_{b}+\sum_{i=1}^{N} \sum_{j>i}^{N} U_{n b, i j}
$$

where $N_{\text {bond }}$ is the number of bonds and $N$ is the number of particles (CG beads) in the system, $U_{b}$ is a bond energy and $U_{n b, i j}$ is a nonbonded interaction potential. We shall now describe the bonded and nonbonded interaction potential in Section 2.2 and 2.3.

\subsection{Bonded potential}

The bonded interaction potential is responsible for maintaining the nanocrystal integrity along the $c$-crystallographic direction. Three potentials were introduced to model the total bonded interaction potential $U_{b}$ of a bond:

$$
U_{b}=U_{h}+U_{a}+U_{d}
$$

where $U_{h}$ and $U_{a}$ are respectively a harmonic bond potential and a cosine harmonic angle, similar to the potentials used by Chen et al. in Ref. 83, and $U_{d}$ is a dihedral potential that we designed. The harmonic bond potential writes

$$
U_{h}=k_{h} r_{c}^{2}
$$

where $k_{h}$ is the spring constant and $r_{c}$ is the ellipsoid contact distance calculated with an effective particle size $r_{h}$, as illustrated in Figure 2(b). It should be noted that this effective particle size can be slightly different than the ellipsoid size and is an adjustable parameter of the force-field. The harmonic bond potential can be rewritten as a function of the effective particle size $r_{h}$, particle to particle vector $\vec{r}=\overrightarrow{r_{i j}}=\overrightarrow{r_{i}}-\overrightarrow{r_{j}}$ and the particles orientation $\left(\hat{e_{i}^{x}}, \hat{e_{i}^{y}}, \hat{e_{i}^{z}}\right),\left(\hat{e_{j}^{x}}, \hat{e_{j}^{y}}, \hat{e_{j}^{z}}\right)$, where $\hat{e_{i}^{x}}$ denotes the unit vector $\overrightarrow{e_{i}^{x}} /\left|\overrightarrow{e_{i}^{x}}\right|$, see Figure $2(\mathrm{~b})$ :

$$
U_{h}=k_{h}\left|\vec{r}+r_{h}\left(\hat{e_{i}^{x}}+\hat{e}_{j}^{x}\right)\right|^{2}
$$


The cosine harmonic angle potential only depends on the particle orientations as illustrated in Figure 2(b) and writes

$$
U_{a}=k_{a}\left[\cos (\theta)-\cos \left(\theta_{0}\right)\right]^{2}
$$

where $k_{a}$ is the bending constant, $\theta$ is the angle between the particles along their x-axis $\left(180^{\circ}\right.$ for the planar configuration showed in Figure $\left.2(\mathrm{~b})\right)$ and $\theta_{0}$ is the equilibrium bond angle. $\theta$ is directly related to the particles orientation by $\cos (\theta)=-\hat{e}_{i}^{x} \cdot e_{j}^{x}$, and thus:

$$
U_{a}=k_{a}\left[\hat{e_{i}^{x}} \cdot \hat{e_{j}^{x}}+\cos \left(\theta_{0}\right)\right]^{2}
$$

The last term in the bonded potential is a dihedral potential aimed at reproducing the twisting observed in CNC. It has indeed been observed that the CNC has a right-handed twist along the $c$-direction induced by the chirality of the saccharide glucose unit, with a rotation of about $1.5^{\circ} / \mathrm{nm}$ for a CNC with 36 chains. ${ }^{84}$ This twist is introduced by the following dihedral potential:

$$
U_{d}=k_{d}\left[1+\cos \left(\phi-\phi_{0}\right)\right]
$$

where $k_{d}$ is the dihedral constant, $\phi$ is the dihedral angle, illustrated in Figure 2(b), and $\phi_{0}$ is the equilibrium angle. The dihedral angle can be expressed as a function of the particle-particle separation and the particles orientation by

$$
\phi=\sigma \arccos (\nu)
$$

with

$$
\sigma=\operatorname{sign}\left[\left(\hat{e}_{j}^{z} \times \vec{r}\right) \cdot \hat{e}_{i}^{z}\right]
$$




$$
\nu=\frac{\left(\vec{r} \times \hat{e_{j}^{z}}\right) \cdot\left(\vec{r} \times \hat{e_{i}^{z}}\right)}{\left|\vec{r} \times \hat{e_{j}^{z}}\right|\left|\vec{r} \times \hat{e_{i}^{z}}\right|}
$$

Every potential $U$ induces forces $\vec{F}$ and torques $\vec{\tau}$ on particle $i$ and $j$ given by: ${ }^{85}$

$$
\begin{aligned}
& \vec{F}=\overrightarrow{F_{i}}=-\overrightarrow{F_{j}}=-\frac{\partial U}{\partial \vec{r}} \\
& \overrightarrow{\tau_{i}}=-\sum_{m=x, y, z} e_{i}^{\hat{m}} \times \frac{\partial U}{\partial e_{i}^{\hat{m}}} \\
& \overrightarrow{\tau_{j}}=-\sum_{m=x, y, z} e_{j}^{\hat{m}} \times \frac{\partial U}{\partial e_{j}^{\hat{m}}}
\end{aligned}
$$

All the final calculated expressions for forces and torques, for all potentials used in the model (including nonbonded potentials presented in the next section), can be found in the Supporting Information.

\subsection{Nonbonded potential}

In our model, we described the nonbonded interactions between two $\mathrm{CNC}$ as interactions between the two CNC surfaces in contact (e.g. interaction between (100)-(100) surfaces, or (110)-(100) surfaces...). These interactions are introduced by decorating the CG beads with six attractive patches, as illustrated in Figure 2(c). But before going into the details of the patches geometrical definition, we shall explain what kind of interaction they represent exactly.

Inspired by the Kern-Frenkel patchy particles model, ${ }^{22} \mathrm{Li}$ and coworkers have introduced a powerful soft-anisotropic potential to describe such surface-anisotropic interactions. ${ }^{76,77}$ This model relies on the introduction of so-called attractive patches at the particles surface, that will interact with each other providing that they are facing each other, and thus promoting a certain relative orientation of two adjacent particles. Although the patches position 
gives an anisotropy to the particle's interaction, the patches by themselves are circular (like in Figure 1), and thus not very suitable to represent real particle facets such as the different surfaces of a CNC. In the following, we introduce a new patch model with an anisotropic spherical rectangle patch shape, see Figure 2(d). This model can be seen as an extension of the model of Li et al., ${ }^{76,77}$ with inspirations from the work of Vacha et al. on patchy spherocylinders. ${ }^{86}$ The nonbonded interaction potential between two particles $i$ and $j$ is the sum of the interactions between all particles patches:

$$
U_{n b}=\sum_{k, l} U_{p, k l}
$$

The interaction between two patches $k$ and $l$, respectively on particles $i$ and $j$, is illustrated in Figure 2(d). Each patch is defined by an orientation (relative to the particle orientation) via the three unit vectors $\left(p_{i, k}^{\hat{x}}, p_{i, k}^{\hat{y}}, p_{i, k}^{\hat{z}}\right)$, and by two aperture angles $\left(\Omega_{i, k}^{x}\right.$, $\left.\Omega_{i, k}^{y}\right)$. The patches interact if they are facing each other, and in this case they will promote alignment and rapprochement of the particles. This is expressed through the following potential:

$$
U_{p, k l}=D_{k, l}(\vec{r}) f_{k, l}\left(p_{i, k}^{\hat{x}}, p_{i, k}^{\hat{y}}, p_{i, k}^{\hat{z}}, p_{j, l}^{\hat{x}}, p_{j, l}^{\hat{y}}, p_{j, l}^{\hat{z}}, \vec{r}\right)
$$

where $D$ is a function depending on the particles distance only, and $f_{k, l}$ is a function containing the angular dependence of the patch-patch interaction. $f_{k, l}$ writes as

$$
\begin{aligned}
& f_{k, l}\left(p_{i, k}^{\hat{x}}, p_{i, k}^{\hat{y}}, p_{i, k}^{\hat{z}}, p_{j, l}^{\hat{x}}, p_{j, l}^{\hat{y}}, p_{j, l}^{\hat{z}}, \vec{r}\right)= \\
& \left\{\begin{array}{ll}
\cos \left(\frac{\pi \theta_{i, k}^{x}}{2 \Omega_{i, k}^{x}}\right) \cos \left(\frac{\pi \theta_{i, k}^{y}}{2 \Omega_{i, k}^{y}}\right) \cos \left(\frac{\pi \theta_{j, l}^{x}}{2 \Omega_{j, l}^{x}}\right) \cos \left(\frac{\pi \theta_{j, l}^{y}}{2 \Omega_{j, l}^{y}}\right) & \text { and } \cos \left(\theta_{i, k}^{y}\right) \geq \cos \left(\Omega_{i, k}^{x}\right) \\
& \text { and } \cos \left(\theta_{j, l}^{x}\right) \geq \cos \left(\Omega_{j, l}^{x}\right) \\
0 & \text { and } \cos \left(\theta_{j, l}^{y}\right) \geq \cos \left(\Omega_{j, l}^{y}\right)
\end{array}\right) \\
& \text { otherwise }
\end{aligned}
$$


where the cosines of the projected angles $\theta_{i, k}^{x}, \theta_{i, k}^{y}, \theta_{j, l}^{x}, \theta_{j, l}^{y}$ are given by

$$
\begin{aligned}
& \cos \left(\theta_{i, k}^{x}\right)=\frac{p_{i, k}^{\hat{z}} \overrightarrow{r_{j i, k}^{x}}}{\left|\overrightarrow{r_{j i, k}^{x}}\right|} \\
& \cos \left(\theta_{i, k}^{y}\right)=\frac{p_{i, k}^{\hat{z}} \overrightarrow{r_{j i, k}^{y}}}{\left|\overrightarrow{r_{j i, k}^{y}}\right|} \\
& \cos \left(\theta_{j, l}^{x}\right)=\frac{p_{j, l}^{\hat{z}} \cdot \overrightarrow{r_{i j, l}^{x}}}{\left|\overrightarrow{r_{i j, l}^{x}}\right|} \\
& \cos \left(\theta_{j, l}^{y}\right)=\frac{p_{j, l}^{\hat{z}} \cdot \overrightarrow{r_{i j, l}^{y}}}{\left|\overrightarrow{r_{i j, l}^{y}}\right|}
\end{aligned}
$$

These cosines depend on the projection of the particle-particle separation $\overrightarrow{r_{i j}}=\vec{r}$ (or $\left.\overrightarrow{r_{j i}}=-\vec{r}\right)$ on the $\left(p^{x}, p^{z}\right)$ and $\left(p^{y}, p^{z}\right)$ planes:

$$
\begin{aligned}
& \overrightarrow{r_{j i, k}^{x}}=\left(p_{i, k}^{\hat{z}} \cdot \overrightarrow{r_{j i}}\right) p_{i, k}^{\hat{z}}+\left(p_{i, k}^{\hat{x}} \cdot \overrightarrow{r_{j i}}\right) p_{i, k}^{\hat{x}} \\
& \overrightarrow{r_{j i, k}^{y}}=\left(p_{i, k}^{\hat{z}} \cdot \overrightarrow{r_{j i}}\right) p_{i, k}^{\hat{z}}+\left(p_{i, k}^{\hat{y}} \cdot \overrightarrow{r_{j i}}\right) p_{i, k}^{\hat{y}} \\
& \overrightarrow{r_{i j, l}^{x}}=\left(p_{j, l}^{\hat{z}} \cdot \overrightarrow{r_{i j}}\right) p_{j, l}^{\hat{z}}+\left(p_{j, l}^{\hat{x}} \cdot \overrightarrow{r_{i j}}\right) p_{j, l}^{\hat{x}} \\
& \overrightarrow{r_{i j, l}^{y}}=\left(p_{j, l}^{\hat{z}} \cdot \overrightarrow{r_{i j}}\right) p_{j, l}^{\hat{z}}+\left(p_{j, l}^{\hat{y}} \cdot \overrightarrow{r_{i j}}\right) p_{j, l}^{\hat{y}}
\end{aligned}
$$

For the distance-dependent function, we found that a Morse potential was a good representation of the CNC surface interactions as it will be shown in section 3.2 , therefore,

$$
D_{k, l}(\vec{r})=U_{M, k, l}\left[\left(1-\exp \left(-a_{M, k, l}\left(|\vec{r}|-r_{M, k, l}\right)\right)\right)^{2}-1\right]
$$

with $U_{M, k, l}$ being the interaction well depth, $r_{M, k, l}$ the equilibrium distance, and $a_{M, k, l}$ 
controlling the width of the potential.

Eventually, to set the patches orientations and geometries shown in Figure 2(c), we started by calculating the edges of the $\mathrm{CG}$ beads by considering the positions of the outer glucose units in the crystal structure. This gives the nearly hexagonal shape on the crosssection of Figure 2(c), or the nearly hexagonal prism on the perspective view. We then divide the cross-section into six circular sectors by considering the angles formed by the particle center-of-mass and the corners of the particle. Finally, the patches y-aperture angles $\Omega^{y}$ and the patches z-orientations $\hat{p}^{z}$ are calculated respectively as the half-angles and bisector of the circular sectors. The patches x-orientations were aligned with the bead orientation $\hat{e}^{x}$ and the patches y-orientations were calculated such that $\left(\hat{p^{x}}, \hat{p^{y}}, \hat{p^{z}}\right)$ form an orthonormal basis. For the patches $\mathrm{x}$-aperture angles, we simply set them to $\Omega^{x}=90^{\circ}$ in order to have a full coverage of the bead with the patches as shown in Figure 2(c). All the geometrical information from the aforementioned procedure are summarized in Table 1.

Table 1: Patches definitions

\begin{tabular}{||ccccccc||}
\hline $\mathrm{n}^{\circ}$ & $\Omega^{y}$ & $\Omega^{x}$ & $\hat{p}^{x}$ & $\hat{p^{y}}$ & $\hat{p}^{z}$ & Type \\
\hline \hline 1 & $22.02^{\circ}$ & $90^{\circ}$ & $(1,0,0)$ & $(0,-0.452,0.892)$ & $(0,0.892,0.452)$ & $(1-10)$ \\
2 & $29.94^{\circ}$ & $90^{\circ}$ & $(1,0,0)$ & $(0,-0.981,0.194)$ & $(0,0.194,0.981)$ & $(100)$ \\
3 & $38.03^{\circ}$ & $90^{\circ}$ & $(1,0,0)$ & $(0,-0.547,-0.837)$ & $(0,-0.837,0.547)$ & $(110)$ \\
4 & $22.02^{\circ}$ & $90^{\circ}$ & $(1,0,0)$ & $(0,0.452,-0.892)$ & $(0,-0.892,-0.452)$ & $(1-10)$ \\
5 & $29.94^{\circ}$ & $90^{\circ}$ & $(1,0,0)$ & $(0,0.981,-0.194)$ & $(0,-0.194,-0.981)$ & $(100)$ \\
6 & $38.03^{\circ}$ & $90^{\circ}$ & $(1,0,0)$ & $(0,0.547,0.837)$ & $(0,0.837,-0.547)$ & $(110)$ \\
\hline
\end{tabular}

\section{Parametrization}

\subsection{Bonded}

We parametrized the bonded interactions developed in the previous section against AAMD simulations of the CNC shown in Figure 2(a) (containing 32 glucose units along the $c$-axis) in water. The computational details of the AA-MD can be found in the Supporting 
Information, and are identical to Ref. 57 since we used exactly the same MD trajectory as in that study. From the equilibrated frames of the AA-MD, we extracted the bond length, bond angle and dihedrals angle distributions of the CG representation of this AA description of the CNC, see the blue histograms in Figure 3. For the extraction of the bonds length, we proceeded in a standard way by computing the center-of-mass distances between the groups of atoms forming adjacent beads in the CG representation (e.g. distance between center-of-mass of all red atoms and center-of-mass of all green atoms in the bottom CNC of Figure 2(a)) and accumulating the values on an histogram for all equilibrated frames, Figure 3(a). For the extraction of the bonds angle and dihedrals angle, we developed a new method to calculate the orientation of the groups of atoms forming adjacent beads. Let us denote $O_{k}$ the point cloud of oxygen atoms forming bead $k$ and $d_{O_{k}, O_{k+1}}=\sum_{i \subset O_{k}, j \subset O_{k+1}}\left|\overrightarrow{r_{i}}-\vec{r}_{j}\right|^{2}$ the distance between two bonded CG beads $k$ and $k+1$. We applied the Iterative Closest Point algorithm (ICP) ${ }^{87}$ to obtain the transformation matrix $\boldsymbol{T}_{k, k+1}$ such that $d_{O_{k}, \boldsymbol{T}\left(O_{k+1}\right)}=$ $\sum_{i \subset O_{k}, j \subset O_{k+1}}\left|\overrightarrow{r_{i}}-\boldsymbol{T}\left(\overrightarrow{r_{j}}\right)\right|^{2}$ is minimized. In other words, we found the transformation $\boldsymbol{T}$ that superimposes the oxygen atoms of bead $k+1$ with the oxygen atoms of bead $k$. This transformation matrix $\boldsymbol{T}$ contains the bonds angle and dihedrals angle, that we accumulated over the equilibrated AA-MD frames to obtain the distributions in Figure 3(b)-(c). More details can be found in the Supporting Information.

After having extracted these distributions, we performed CG-MD simulations of one CNC containing four beads. The simulations were carried out with a modified version of the LAMMPS simulation package, in an implicit solvent, with the nve/asphere integrator and a Langevin thermostat with a damping factor of $4.0 \mathrm{ps}$. The time step was set to 100 fs and the temperature to $300 \mathrm{~K}$. The same conditions were applied throughout the entire study. Note that to study the dynamics of the single CNC, periodic boundary conditions were applied in xyz directions, and the simulation box was chosen big enough so that the CNC cannot interact with itself across the boundaries, just like for the AA-MD simulation. The distribution of bonds length, bonds angle and dihedrals angle were extracted on equilibrated 
(a)

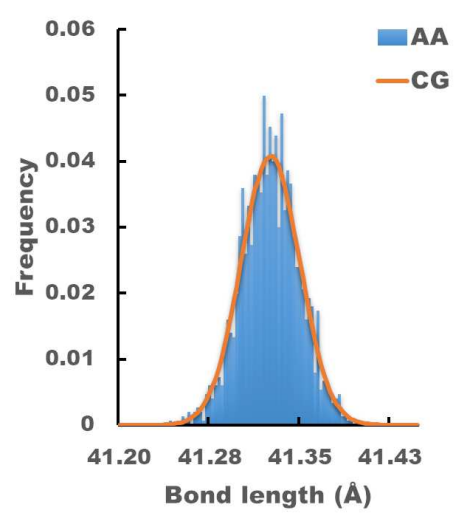

(b)

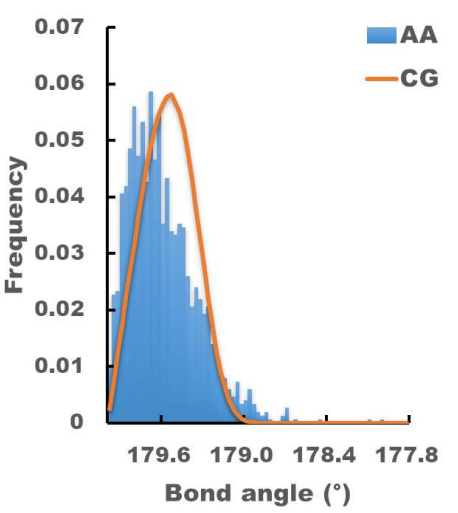

(c)

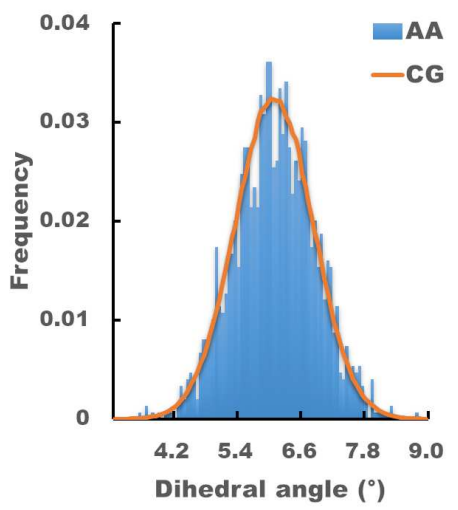

Figure 3: Parametrization of the bonded CG force-field. The plots show the distributions of bonds length, bonds angle and dihedrals angle obtained from AA-MD and from CG-MD after proper parametrization.

frames of these MD simulations, and we adjusted the force-field parameters introduced in the previous section until the distributions from AA-MD and CG-MD superimpose. We finally obtained the satisfactory agreement shown in Figure 3 for the set of parameters reported in Table 2.

Table 2: Bonded force-field parameters

\begin{tabular}{||ccc||}
\hline Bonded interaction & Force constant & Equilibrium value \\
\hline \hline Harmonic bond $U_{b}$ & $k_{b}=500 \mathrm{kcal} / \mathrm{mol} / \AA^{2}$ & $r_{h}=20.66 \AA$ \\
Cosine harmonic angle $U_{a}$ & $k_{a}=15 \times 10^{7} \mathrm{kcal} / \mathrm{mol}$ & $\theta_{0}=180^{\circ}$ \\
Dihedral $U_{d}$ & $k_{d}=3600 \mathrm{kcal} / \mathrm{mol}$ & $\phi_{0}=6.1^{\circ}$ \\
\hline
\end{tabular}

\subsection{Nonbonded}

We parametrized the Morse potential appearing in the patch-patch interaction in Equation 22 against AA-MD simulations following the method in Ref. 57. The Potential of Mean Force (PMF) for AA-MD simulations of two CNC lying parallel to each other, in water, were calculated for different surface interactions as depicted by the CNC cross-sections in Figure 4. The periodic boundary conditions were maintained in xyz directions, and the box dimension were big enough in y and $\mathrm{z}$ directions such that the two CNC interact only with each other's 
in the central box (not across the boundaries). However, the CNC cross the $\mathrm{x}$-boundary along their c-axis such that we are simulating infinitely long CNC. Therefore, the obtained PMF are a bit biased because the twist is prohibited, but since the situation is comparable both for AA and CG the comparison is still valid. On the other hand, this setup has the advantage to remove effects of the $\mathrm{CNC}$ end, and it prevents the rotation of the $\mathrm{CNC}$ during the simulation towards their preferred surface interaction. More information can be found in the Supporting Information. We used umbrella sampling simulations to calculate the PMF as it was done previously by Paajanen and coworkers. ${ }^{45}$ Two CNC were first put in contact without water between them, one was restrained to its position, and a pulling force was then applied to the other in order to generate several CNC configurations at different surface-surface distances. For each configuration, an AA-MD was conducted and the PMF extracted using the Weighted Histogram Analysis Method (WHAM). We refer the reader to Ref. 57 for the computational details. This procedure provides the AA PMF curves of Figure 4 for different surface-surface interactions as a function of the surface-surface distance. The standard deviation, as obtained from the BOOTSTRAP algorithm implemented in GROMACS, ${ }^{88}$ was approximately $2 \%$. We conducted the same PMF calculations for our new CG model, and adjusted the Morse potential parameters in order to superimpose the AA PMF and the CG PMF. As it can be seen on Figure 4, we obtained an excellent agreement for the set of parameters reported in Table 3. Note that the PMF were scaled by the number of glucose units for each surface, and we were therefore able to parametrize all possible surface-surface interactions from the three configurations presented in Figure 4. For all patchy interactions the distance cut-off was set to $10 \mathrm{~nm}$ and we excluded these interactions for bonded particles up to the third bond.

It is worth noting that in the present model we needed three different patches to model the three different CNC surfaces while in our previous CG model of the same CNC, Ref. 57, only two beads were used: One for the (100) surface and one for the (110) and (1-10) surfaces. We could do that previously because we used "classical" CG beads interacting with Lennard- 
(a)

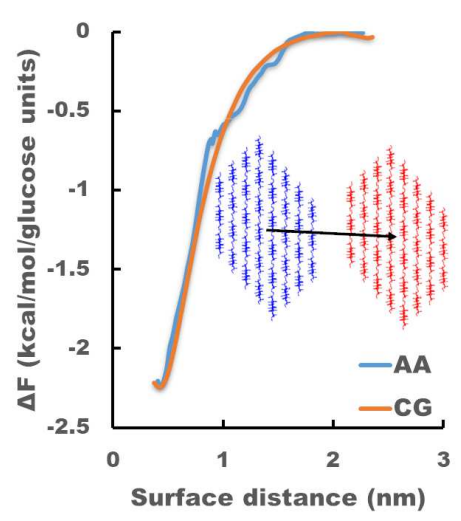

(b)

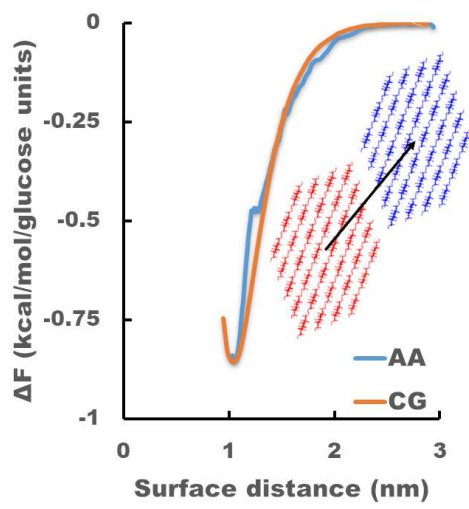

(c) $\quad(1-10)-(100)$

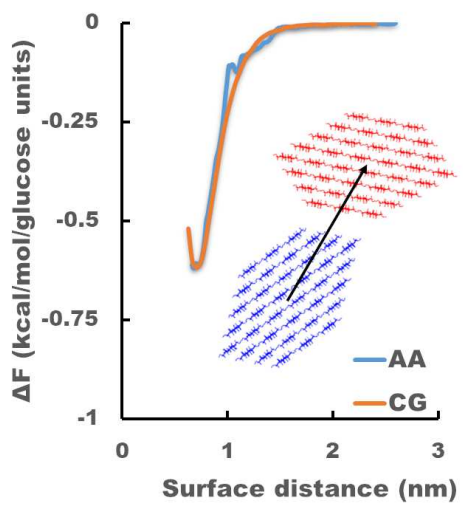

Figure 4: Parametrization of the nonbonded CG force-field. The plots show the PMF curves calculated for various surface-surface interactions, both from AA-MD and CG-MD after proper parametrization. The configurations of the two CNC used to calculate the PMF are schematically depicted by the blue and red CNC cross-sections.

Table 3: Surface-surface interactions

\begin{tabular}{||cccc||}
\hline Surfaces & $U_{M, k, l}(\mathrm{Kcal} / \mathrm{mol})$ & $a_{M, k, l}\left(\AA^{-1}\right)$ & $r_{M, k, l}(\AA)$ \\
\hline \hline$(100)-(100)$ & 110.0 & 0.326 & 31.26 \\
$(100)-(110)$ & 40.9 & 0.55 & 33.88 \\
$(100)-(1-10)$ & 35.8 & 0.55 & 38.45 \\
$(110)-(110)$ & 70.0 & 0.4 & 37.0 \\
$(110)-(1-10)$ & 63.0 & 0.4 & 41.57 \\
$(1-10)-(1-10)$ & 56.0 & 0.4 & 46.15 \\
\hline
\end{tabular}

Jones potential, and therefore: (i) the beads for (110) and (1-10) not only represented the surface glucose units but very similar groups of glucose units including inner-CNC glucose units, (ii) the non-regular shape of the CNC cross-section could be reproduced by setting different positions of the (110) and (1-10) beads around the CNC core, (iii) since the surface interactions arose from all beads interacting at the same time, the $\mathrm{CNC}-\mathrm{CNC}$ interaction was different for surfaces (110) and (1-10) because of the position of these beads around the core (even though the beads themselves were identical). In the patchy particle model, since the surface-surface interactions only arose from one single patch-patch interaction at a time, we were obliged to have different patches for (110) and (1-10) surfaces in order to have a non-regular CNC shape and different interactions for surfaces (110) and (1-10). The physical 
meaning of the parameters reported in Table 3 for the various surfaces can be understood by considering the hydrophobic or hydrophilic properties of the surface: the (100) surface exposes $\mathrm{C}-\mathrm{H}$ moieties to the surrounding medium and therefore is hydrophobic, while the (110) and (1-10) are decorated by protruding hydroxyls and are hydrophilic. ${ }^{89}$ Since the values for $U_{M, k, l}$ include the effect of different surface areas (i.e. number of glucose units at the surface) and the values for $r_{M, k, l}$ include the effect of different CNC core to surface distances, it is more straightforward to directly inspect the PMF of Figure 4, from which they are derived. The interaction is the strongest for (100)-(100) surfaces, with the lowest equilibrium distance. The two surfaces being hydrophobic, at equilibrium there is no layer of water between the $\mathrm{CNC}$, they are very close and interact strongly via direct Van der Walls interactions. The (110)-(110) hydrophilic surfaces have a weaker interaction because at equilibrium there is one or more layers of water in between the CNC that reduce the direct Van der Walls interactions. Therefore they also exhibit the largest equilibrium distance. Finally, the equilibrium distance between the hydrophobic (100) surface and the hydrophilic (1-10) surface is intermediate because in this case there is a competition between the hydrophobic surface that tends to expel the water in between the $\mathrm{CNC}$ and the hydrophilic surface that tends to retain the water. As a results, the interaction between these surfaces is the weaker because the hydrophilic and hydrophobic properties of the two surfaces in contact cannot

be meet concomitantly. Finally, the parameter $a_{M, k, l}$ essentially replicates the information contained in $U_{M, k, l}$ since for the Morse potential $a_{M, k, l} \propto 1 / \sqrt{2 U_{M, k, l}}$.

\section{Applications}

\subsection{Single nanocrystal properties}

To assess the validity of the bonded parametrization, we calculated the longitudinal elastic modulus $E$ of a $\mathrm{CNC}$ along the $c$-direction as well as the diffusion coefficient of the $\mathrm{CNC}$ as a function the CNC length. To perform these simulations, periodic boundary conditions 
were applied in xyz directions and the boxes were big enough to fit in the CNC with desired dimensions. Yet, the $\mathrm{CNC}$ could in principle interact with itself across the boundaries, or with other $\mathrm{CNC}$ in the case of diffusion, but we turned-off the non-bonded interactions since we were interested in properties depending on the bonded interactions only. $E$ was obtained by a steered MD simulation of a single CNC of length $\sim 200 \mathrm{~nm}$ when the position of one end of the CNC was fixed and a gradual pulling force was applied to the other end. We monitored the CNC elongation from this dynamics and we extracted the Stress-Strain curve shown in Figure 5(a). The slope of the curve gives us an elastic modulus $E=340 \mathrm{GPa}$. Note that in our CG model, the cross-sectional area (used to calculate the stress) remains constant when the CNC is steered. AA-MD performed by Poma et al. on the same model of CNC show that this is actually the expected behavior. ${ }^{55}$ The calculated value is close to those reported in the literature, both theoretical and experimental, which cover a large range $(50 \sim 220$ GPa) depending on the CNC source, extraction method and cross-sectional morphology. ${ }^{90}$ In particular, this value, if scaled by the number of chains in the CNC, i.e. $340 \times(18 / 36)=$ $170 \mathrm{GPa}$, is quite close to the elastic modulus $E=161 \mathrm{GPa}$ found theoretically by Muthoka et al. for the same CNC cross-section morphology but with 18 chains. ${ }^{91}$ Note that in our previous supra-CG model of $\mathrm{CNC}$, the elastic modulus $E=110 \mathrm{GPa}$ was somehow lower. ${ }^{57}$

The diffusion coefficient was estimated for three different CNC lengths, $8 \mathrm{~nm}, 200 \mathrm{~nm}$ and $400 \mathrm{~nm}$, by monitoring the Mean Squared Displacement (MSD) of 200 non-interacting $\mathrm{CNC}$ in a simulation box during $7 \mu \mathrm{s}$. The results are shown in Figure 5(b), and are in good agreement with experimental data as well as theoretical calculations from our previous supra-CG model. ${ }^{57}$

\subsection{Cholesteric phase of the cellulose nanocrystal}

In this section, we finally test the ability of this new patchy particle model to reproduce the self-assembly of CNC into a so-called cholesteric phase. Note that in all the simulations presented in this section, periodic boundary conditions were applied in xyz directions. Yet, 
(a)

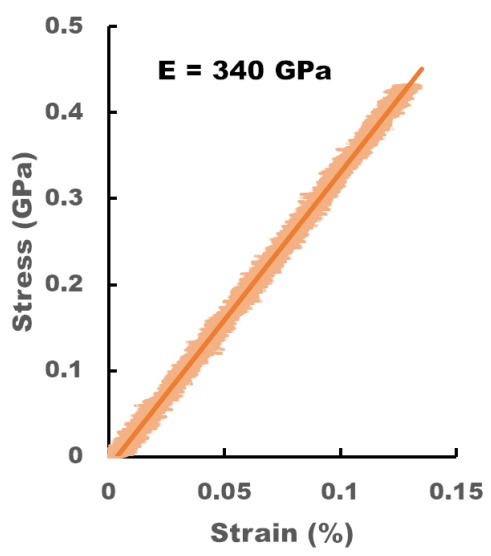

(b)

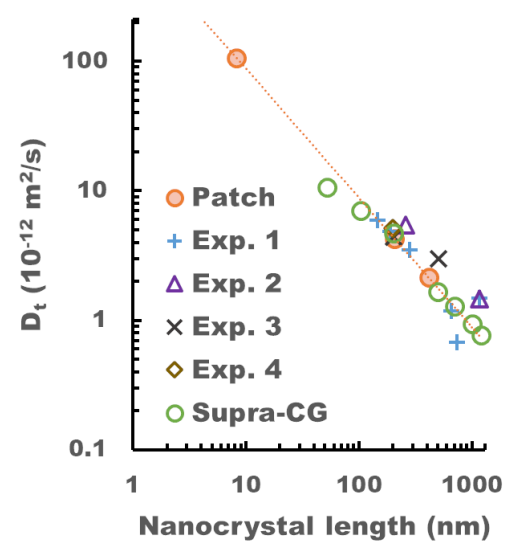

Figure 5: (a) Stress-Strain curve obtained from CG-MD simulation and extracted value of the longitudinal elastic modulus. (b) CNC diffusion coefficient as a function of the CNC length. Values from the present patch model are represented as orange circles with a linear fit superimpose (orange dotted line). Experimental values come from Ref. 92 for Exp. 1, Ref. 93 for Exp. 2, Ref. 94 for Exp. 3, Ref. 95 for Exp. 4. The set of points denoted Supra-CG correspond to simulation results from our previous supra-CG model of CNC, see Ref. 57.

the simulation boxes were chosen big enough so that there were no interactions across the boundaries, and the presented systems can be seen as isolated systems. We start by simulating the equilibration of a model system made up of a long layer of $180 \mathrm{CNC}$ put next to each other in a parallel alignment, with the (110) surfaces facing each other, see Figure 6(a). This structure was simulated for different CNC lengths, from $8 \mathrm{~nm}$ to $100 \mathrm{~nm}$, and we ran the dynamics until an equilibrated state was reached as indicated by a plateau in the MD energy curve. The equilibrated structures form a left-handed helical ribbon where the CNC c-directions are rotating in the $(\mathrm{x}-\mathrm{z})$ planes, as shown in $6(\mathrm{a})$ for the $\mathrm{CNC}$ length $\sim 41.5 \mathrm{~nm}$. It proves the ability of the model to reproduce the tendency of the right-handed $\mathrm{CNC}$ to form a left-handed cholesteric phase due to their intrinsic twist. ${ }^{96}$ It is worth noting that in our previous supra-CG model of $\mathrm{CNC}$, we were not able to directly simulate this cholesteric ordering due to computational limits. This qualitatively indicates better performances of this new patchy particle model regarding the simulation of cholesteric ordering, and we plan to 
provide a quantitative comparison in a future study. We also foresee that the implementation of our model with GPU acceleration would bring significant speedup considering for instance that Li et al. have reported a speedup of two orders of magnitude between LAMMPS with one CPU and GALAMOST ${ }^{97,98}$ with one GPU for a benchmark system with 40000 ellipsoid particles. ${ }^{77}$ We measured the pitch $p$ (y-distance such that the $c$-directions has rotated by $360^{\circ}$ in the (x-z) plane) of these cholesteric ribbons as a function of the CNC length, see Figure 6(b). While this study cannot be directly compared quantitatively to experimental data, because CNC form 3D cholesteric phases instead of 1D ribbons and because the surface modification have a crucial role in determining the cholesteric pitch, the results are in qualitative agreement with the findings of Beck-Candanedo et al. ${ }^{99}$ They found that for spruce-derived CNC at a constant mass fraction in solution, the pitch increases from $7 \mu \mathrm{m}$ to $18 \mu \mathrm{m}$ when the average length of the $\mathrm{CNC}$ increases from $\sim 100 \mathrm{~nm}$ to $\sim 140 \mathrm{~nm}$. There was no clear explanation of this effect in the original paper, but later on Honorato-Rios and coworkers commented the experiment: 100 "At present this is a purely empirical observation without any proposed explanation for the effect. One may speculate that the greater rod length affects the elastic constants of the phase, rendering twist less favorable [...]". Indeed our results can be qualitatively understood in terms of the energy stored in a twisted ribbon. A very complete mathematical model has been developed by Chopin and coworkers to describe the morphology of a ribbon subjected to twist. ${ }^{101,102}$ The analytical treatment is rather complex, but essentially relies on the fact that twisting a ribbon to form an helicoid induces a longitudinal compressive stress (in the $y$-direction) because the edges of the helix are much longer that the central part of it, and that when this stress is too high it can be relaxed by forming longitudinal or transverse wrinkling, loops or self-contact zones. In our case, there is a competition between the tendency of the ribbon to twist toward a prescribe angle due to the intrinsic twist of the $\mathrm{CNC}$, and the apparition of a compressive stress that tends to untwist the ribbon. The larger the ribbon width the larger the compressive stress, and therefore the pitch increases (the ribbon tends to untwist) when the CNC length (the 
ribbon width) increases. When the $\mathrm{CNC}$ length reaches $100 \mathrm{~nm}$, there is the apparition of longitudinal wrinkles with a wavelength $\lambda$ as shown in Figure 6(c), in agreement with the theoretical and experimental findings of Chopin et al. This wrinkling reduces the effective length of the ribbon and therefore the pitch increase with the CNC length is less pronounced at $100 \mathrm{~nm}$ than for shorter lengths. While this model 1D cholesteric ribbon is not representative of the real cholesteric phase morphology of $\mathrm{CNC}$, it can be of significant importance for a wide variety of other building blocks such as amphiphilic lipids or proteins that can assemble into such nanoribbons, ${ }^{103}$ opening up a large field of applications for our new patchy particles model.

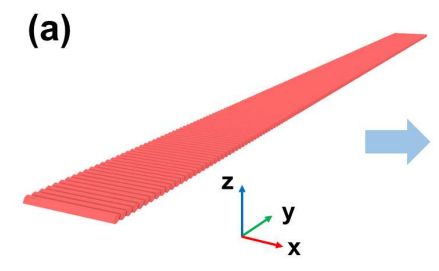

(b)

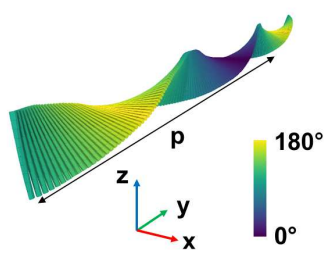

(c)
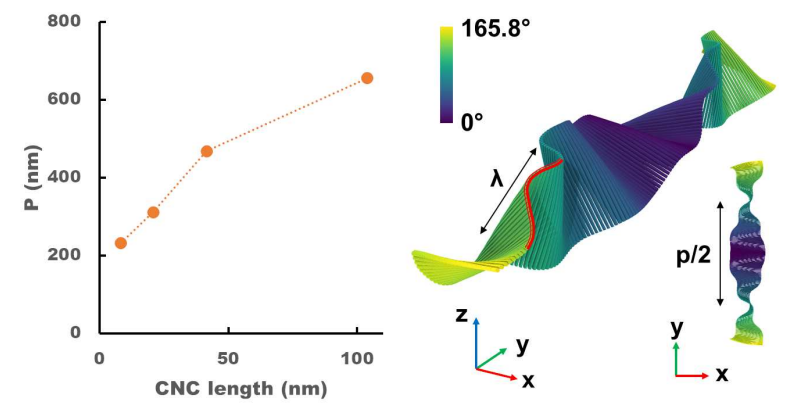

Figure 6: (a) Initial model structure with $180 \mathrm{CNC}$ of length $\sim 41.5 \mathrm{~nm}$ side-by-side with (110) surfaces facing each other, and corresponding equilibrated cholesteric ribbon. The CG beads are colored according to the angle between their $\hat{e_{x}}$ axis and the $x$-axis, and the cholesteric pitch $p$ is highlighted. (b) Pitch as a function of the CNC length. Orange dotted line is a guide for the eyes. (c) Equilibrated cholesteric ribbon for CNC length $100 \mathrm{~nm}$. Longitudinal undulations of wavelength $\lambda$ are visible. The software package OVITO was used for visualization. ${ }^{104}$

As stated previously, CNC self-assemble into 3D cholesteric phase in solution, when the solution undergoes a phase separation between an isotropic liquid crystal phase and the anisotropic cholesteric phase during the solvent evaporation. The first stage of the phase separation, when the cholesteric phase nucleates into ellipsoidal nanodroplets, the so-called 
tactoids, has a significant role for the rest of the evolution of the cholesteric phase, up to the

final film after drying. ${ }^{105}$ We investigated the cholesteric ordering starting from an already formed compact nanodroplet as illustrated in Figure 7(a). This model system comprises an array of $20 \times 20 \mathrm{CNC}$ of length $\sim 83 \mathrm{~nm}$ arranged into a hexagonal structure. We let the system relax to its equilibrated state as shown on the right in Figure 7(a), which took about 10 ns. The black lines outline the nanodroplet contour, highlighting the right-handed twist that develops along the $x$-axis. A closer inspection of the CNC orientation in the $(\mathrm{y}, \mathrm{z})$ plane shows an onion-like concentric pattern, where the $\mathrm{CNC}$ attempt to radially twist (the helicoidal axes are along the radii of the circular rings). This particular structure, known as a non-singular $\lambda^{+1}$ cholesteric disclination, has been both modelled theoretically ${ }^{106}$ and observed experimentally ${ }^{107}$ for liquid crystals, and is the result of an orientational frustration experienced by the $\mathrm{CNC}$ at the center of the nanodroplet. As a result, left-handed cholesteric order appears in the three surface interaction directions, (110), (100) and (1-10), as illustrated in Figure 7(b). Remarkably, the extent of the twisting in the tactoid along the 3 directions (about $20-25^{\circ}$ as highlighted by the colorbar) is rather similar even though the corresponding surfaces have different interaction strengths and equilibrium distances. Actually, a more precise analysis of the tactoid structure reveals that the concentric pattern consists of circular layers separated by $3.2 \mathrm{~nm}$, and $\mathrm{CNC}$ from one layer to the next have radially twisted of $0.3^{\circ}$, giving a radial pitch of $1.2 \mu \mathrm{m}$ similar in all directions. This constant radial pitch is consistent with the perfect bright circles observed experimentally on tactoid with polarized optical microscopy.

\section{Conclusion}

In summary, a new patchy particle model for molecular dynamics simulations has been developed. Compared to existing models, it provides more flexibility by replacing the circular patch geometry by a spherical rectangle patch geometry, which is well-suited to model 


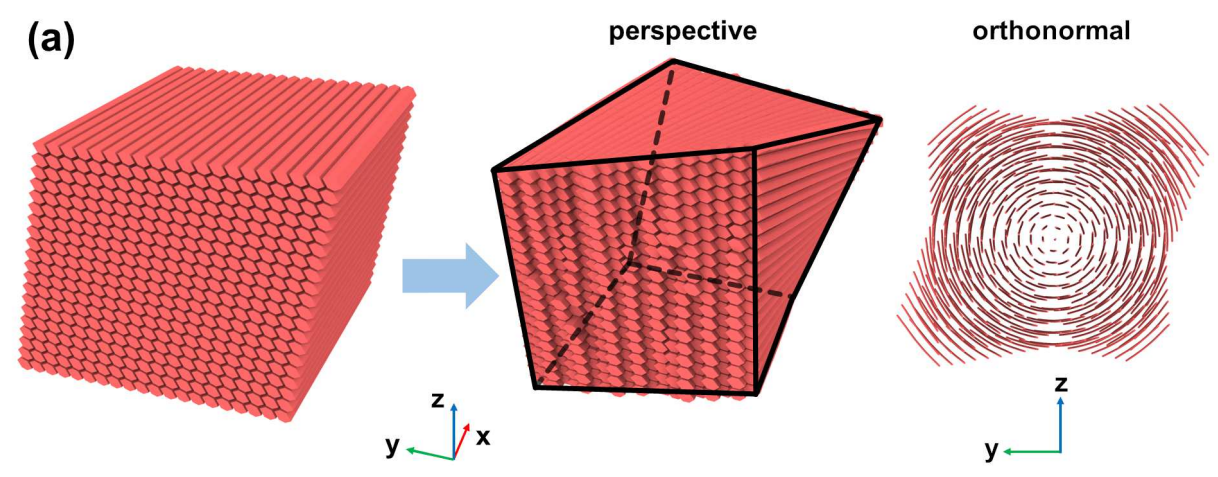

(b)
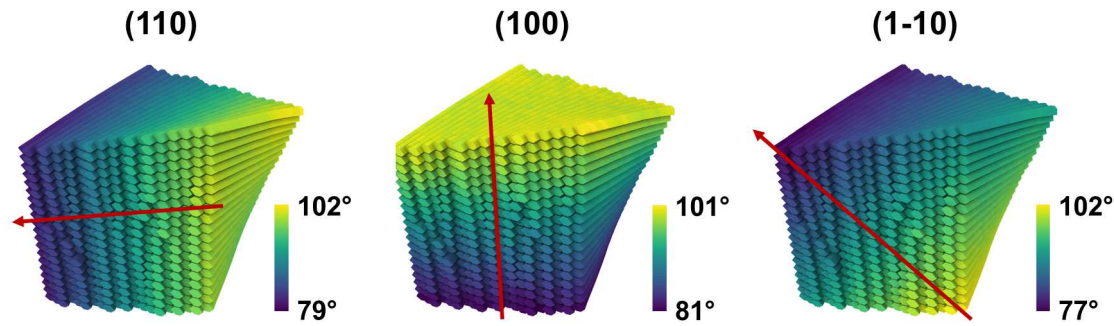

Figure 7: (a) Initial model structure of a CNC tactoid, containing 400 CNC of length $\sim 83$ $\mathrm{nm}$, and relaxed structure in perspective and orthonormal view. For the perspective view, black lines outline the tactoid shape to help visualize the global right-handed twist. For the orthonormal view, CNC are represented as thin rods in order to show the concentric pattern. (b) Cholesteric ordering in three different directions corresponding to the three surfacesurface interactions. The red arrows show the directions of the surface-surface interactions. $\mathrm{Cg}$ beads are colored according to the angle between their $\hat{e_{x}}$ orientation and the z-axis for (110), the y-axis for (100), and the vector $\vec{y}+\vec{z}$ for (1-10). The software package OVITO was used for visualization. ${ }^{104}$ 
faceted particles for instance. It opens the way to investigate self-assembly at large space and time scales in a broad range of systems. This is demonstrated by the development of a new supra coarse-grained model of cellulose nanocrystal where the interactions between the surfaces of distinct nanocrystal are introduced by patchy interactions. The coarse-grained model is parametrized against the results of all-atoms molecular dynamics simulations of the nanocrystal and the validity of the approach is demonstrated by calculating the longitudinal elastic modulus and the diffusion coefficient of a single nanocrystal. The cholesteric ordering of the cellulose is investigated on two model systems. First, cholesteric ribbons are simulated and the dependence of the cholesteric pitch with different nanocrystal length is discussed. The trend is explained considering the elastic energy stored in the structure and for the first time the occurrence of longitudinal undulations in a twisted ribbons are reported at the molecular scale, an effect that has been theoretically predicted and experimentally observed in macroscale ribbons. A cholesteric nanodroplet, a so-called tactoid, is then simulated and demonstrates the potential of the model to investigate the nucleation step during phase separation of the cellulose nanocrystal suspension into a cholesteric phase. In particular, the formation of a non-singular $\lambda^{+1}$ cholesteric disclination is reported, in agreement with the literature. Having such insights into the morphology of the CNC cholesteric nanodroplet is instrumental for the interpretation of the optical properties of the material but the full description of the CNC tactoids will require to include surface charges and solvent ionic strength effects, a work that we are performing currently. Indeed, these parameters can drastically modify the thermodynamics of an assembly of rod-like molecules forming cholesteric phase, and as a result strongly affect the cholesteric pitch. ${ }^{108}$ Yet, the two cholesteric model systems introduced in this study show that this new patchy particle model provides a solid base for the systematic investigation of the interplay between microscopic properties of the molecules and macroscopic properties of the ordered phase such as the cholesteric pitch. The general model presented in this paper, including the extension of the Kern-Frenkel patchy particle model but also the derivation of the bonded force-field for chiral molecules, can be applied 
to any rod-like molecules. All what is required is to perform the parametrization against All-Atom Molecular Dynamics, for which we have detailed all necessary steps. Starting from an isotropic distribution of rod-like molecules, we envisage that the model will be able to investigate the full phase transformation towards the cholesteric phase, giving access to the cholesteric volume fraction, the cholesteric pitch, and the potential presence of defects. As a result, it could be used to design the system to obtain a targeted final morphology or to help the interpretation of experimental results such as polarized light microscopy. For the future, we plan to extend the model in order to account for surface modification of the cellulose nanocrystal as well as to investigate different cellulose nanocrystal cross-sectional shape, and we also envisage to develop GPU implementation of the new patchy particles model.

\section{Acknowledgement}

This work was supported by the Swedish Research Council (Projects 2016-05990), Peter Wallenberg Foundation, Troëdsson foundation, Digital Cellulose Center. IZ thanks the Advanced Functional Material Center at Linköping University for support. ML thanks SeRC (Swedish e-Science Research Center) for funding. IZ acknowledge the support of Wallenberg Wood Science Center. The computations were performed on resources provided by the Swedish National Infrastructure for Computing (SNIC) at NSC and HPC2N.

\section{Supporting Information Available}

The following files are available free of charge.

- SI.pdf: Forces and torques expressions for all potentials used in the model. Details on All-Atom Molecular Dynamics simulations. Link to our modified version of LAMMPS and scripts to set up / run a cholesteric ribbon system. 
- cholesteric_ribbon_N10.avi: Movie of the cholesteric ribbon simulation with CNC length $\sim 41.5 \mathrm{~nm}$. The software package OVITO was used for visualization. ${ }^{104}$

\section{References}

(1) Mendes, A. C.; Baran, E. T.; Reis, R. L.; Azevedo, H. S. Self-assembly in nature: using the principles of nature to create complex nanobiomaterials. Wiley Interdiscip Rev Nanomed Nanobiotechnol 2013, 5, 582-612.

(2) Hacohen, A.; Hanniel, I.; Nikulshin, Y.; Wolfus, S.; Abu-Horowitz, A.; Bachelet, I. Meshing complex macro-scale objects into self-assembling bricks. Scientific Reports 2015, 5, 1-8.

(3) Pawar, A. B.; Kretzschmar, I. Fabrication, Assembly, and Application of Patchy Particles. Macromolecular Rapid Communications 2010, 31, 150-168.

(4) Sacanna, S.; Korpics, M.; Rodriguez, K.; Colón-Meléndez, L.; Kim, S.-H.; Pine, D. J.; Yi, G.-R. Shaping colloids for self-assembly. Nature Communications 2013, 4, 1-6.

(5) Morphew, D.; Shaw, J.; Avins, C.; Chakrabarti, D. Programming Hierarchical SelfAssembly of Patchy Particles into Colloidal Crystals via Colloidal Molecules. ACS Nano 2018, 12, 2355-2364.

(6) Duguet, E.; Hubert, C.; Chomette, C.; Perro, A.; Ravaine, S. Patchy colloidal particles for programmed self-assembly. Comptes Rendus Chimie 2016, 19, 173-182.

(7) Chapela, G. A.; Guzmán, O.; Martínez-González, J. A.; Díaz-Leyva, P.; QuintanaH, J. Self-assembly of kagome lattices, entangled webs and linear fibers with vibrating patchy particles in two dimensions. Soft Matter 2014, 10, 9167-9176.

(8) Romano, F.; Sciortino, F. Patchy from the bottom up. Nature Materials 2011, 10, $171-173$. 
(9) Romano, F.; Sciortino, F. Two dimensional assembly of triblock Janus particles into crystal phases in the two bond per patch limit. Soft Matter 2011, 7, 5799-5804.

(10) Chen, Q.; Diesel, E.; Whitmer, J. K.; Bae, S. C.; Luijten, E.; Granick, S. Triblock Colloids for Directed Self-Assembly. J. Am. Chem. Soc. 2011, 133, 7725-7727.

(11) Chen, Q.; Bae, S. C.; Granick, S. Directed self-assembly of a colloidal kagome lattice. Nature 2011, 469, 381-384.

(12) Romano, F.; Sciortino, F. Patterning symmetry in the rational design of colloidal crystals. Nature Communications 2012, 3, 1-6.

(13) Mao, X.; Chen, Q.; Granick, S. Entropy favours open colloidal lattices. Nature Materials 2013, 12, 217-222.

(14) Mao, X. Entropic effects in the self-assembly of open lattices from patchy particles. Phys. Rev. E 2013, 87, 062319.

(15) Cates, M. E. Entropy stabilizes open crystals. Nature Materials 2013, 12, 179-180.

(16) Chen, Q.; Bae, S. C.; Granick, S. Staged Self-Assembly of Colloidal Metastructures. J. Am. Chem. Soc. 2012, 134, 11080-11083.

(17) Guo, R.; Mao, J.; Xie, X.-M.; Yan, L.-T. Predictive supracolloidal helices from patchy particles. Scientific Reports 2014, 4, 1-7.

(18) Fejer, S. N.; Chakrabarti, D.; Kusumaatmaja, H.; Wales, D. J. Design principles for Bernal spirals and helices with tunable pitch. Nanoscale 2014, 6, 9448-9456.

(19) Olesen, S. W.; Fejer, S. N.; Chakrabarti, D.; Wales, D. J. A left-handed building block self-assembles into right- and left-handed helices. RSC Adv. 2013, 3, 12905-12908.

(20) Morgan, J. W. R.; Chakrabarti, D.; Dorsaz, N.; Wales, D. J. Designing a Bernal Spiral from Patchy Colloids. ACS Nano 2013, 7, 1246-1256. 
(21) Chen, Q.; Whitmer, J. K.; Jiang, S.; Bae, S. C.; Luijten, E.; Granick, S. Supracolloidal Reaction Kinetics of Janus Spheres. Science 2011, 331, 199-202.

(22) Kern, N.; Frenkel, D. Fluid-fluid coexistence in colloidal systems with short-ranged strongly directional attraction. J. Chem. Phys. 2003, 118, 9882-9889.

(23) Noya, E. G.; Vega, C.; Doye, J. P. K.; Louis, A. A. The stability of a crystal with diamond structure for patchy particles with tetrahedral symmetry. J. Chem. Phys. 2010, 132, 234511.

(24) Romano, F.; Russo, J.; Tanaka, H. Influence of Patch-Size Variability on the Crystallization of Tetrahedral Patchy Particles. Phys. Rev. Lett. 2014, 113, 138303.

(25) Romano, F.; Sanz, E.; Sciortino, F. Role of the Range in the Fluid-Crystal Coexistence for a Patchy Particle Model. J. Phys. Chem. B 2009, 113, 15133-15136.

(26) Smallenburg, F.; Sciortino, F. Liquids more stable than crystals in particles with limited valence and flexible bonds. Nature Physics 2013, 9, 554-558.

(27) García, N. A.; Gnan, N.; Zaccarelli, E. Effective potentials induced by self-assembly of patchy particles. Soft Matter 2017, 13, 6051-6058.

(28) Romano, F.; Sanz, E.; Sciortino, F. Crystallization of tetrahedral patchy particles in silico. J. Chem. Phys. 2011, 134, 174502.

(29) Romano, F.; Sanz, E.; Sciortino, F. Phase diagram of a tetrahedral patchy particle model for different interaction ranges. J. Chem. Phys. 2010, 132, 184501.

(30) Lawson, J. L.; Jenness, N. J.; Clark, R. L. Optical trapping performance of dielectricmetallic patchy particles. Opt Express 2015, 23, 33956-33969.

(31) McConnell, M. D.; Kraeutler, M. J.; Yang, S.; Composto, R. J. Patchy and Multiregion Janus Particles with Tunable Optical Properties. Nano Lett. 2010, 10, 603-609. 
(32) Hu, J.; Zhou, S.; Sun, Y.; Fang, X.; Wu, L. Fabrication, properties and applications of Janus particles. Chem. Soc. Rev. 2012, 41, 4356-4378.

(33) Durand, G. In Polymers, Liquid Crystals, and Low-Dimensional Solids; March, N., Tosi, M., Eds.; Physics of Solids and Liquids; Springer US: Boston, MA, 1984; pp $239-285$.

(34) Gennes, P. G.; Prost, J. The Physics of Liquid Crystals; Clarendon Press, 1993.

(35) Chilaya, G. In Chirality in Liquid Crystals; Kitzerow, H.-S., Bahr, C., Eds.; Partially Ordered Systems; Springer New York: New York, NY, 2001; pp 159-185.

(36) Klemm, D.; Kramer, F.; Moritz, S.; Lindström, T.; Ankerfors, M.; Gray, D.; Dorris, A. Nanocelluloses: A New Family of Nature-Based Materials. Angewandte Chemie International Edition 2011, 50, 5438-5466.

(37) Azizi Samir, M. A. S.; Alloin, F.; Dufresne, A. Review of Recent Research into Cellulosic Whiskers, Their Properties and Their Application in Nanocomposite Field. Biomacromolecules 2005, 6, 612-626.

(38) Honorato-Rios, C.; Kuhnhold, A.; Bruckner, J. R.; Dannert, R.; Schilling, T.; Lagerwall, J. P. F. Equilibrium Liquid Crystal Phase Diagrams and Detection of Kinetic Arrest in Cellulose Nanocrystal Suspensions. Front. Mater. 2016, 3.

(39) Paajanen, A.; Ceccherini, S.; Maloney, T.; Ketoja, J. A. Chirality and bound water in the hierarchical cellulose structure. Cellulose 2019, 26, 5877-5892.

(40) Djahedi, C.; Berglund, L. A.; Wohlert, J. Molecular deformation mechanisms in cellulose allomorphs and the role of hydrogen bonds. Carbohydr Polym 2015, 130, 175-182.

(41) Oehme, D. P.; Downton, M. T.; Doblin, M. S.; Wagner, J.; Gidley, M. J.; Bacic, A. Unique aspects of the structure and dynamics of elementary $\mathrm{I} \beta$ cellulose microfibrils revealed by computational simulations. Plant Physiol. 2015, 168, 3-17. 
(42) Conley, K.; Godbout, L.; Whitehead, M. A. T.; van de Ven, T. G. M. Origin of the twist of cellulosic materials. Carbohydrate Polymers 2016, 135, 285-299.

(43) Matthews, J. F.; Skopec, C. E.; Mason, P. E.; Zuccato, P.; Torget, R. W.; Sugiyama, J.; Himmel, M. E.; Brady, J. W. Computer simulation studies of microcrystalline cellulose I $\beta$. Carbohydr. Res. 2006, 341, 138-152.

(44) Chundawat, S. P. S.; Bellesia, G.; Uppugundla, N.; da Costa Sousa, L.; Gao, D.; Cheh, A. M.; Agarwal, U. P.; Bianchetti, C. M.; Phillips, G. N.; Langan, P.; Balan, V.; Gnanakaran, S.; Dale, B. E. Restructuring the Crystalline Cellulose Hydrogen Bond Network Enhances Its Depolymerization Rate. J. Am. Chem. Soc. 2011, 133, 1116311174.

(45) Paajanen, A.; Sonavane, Y.; Ignasiak, D.; Ketoja, J. A.; Maloney, T.; Paavilainen, S. Atomistic molecular dynamics simulations on the interaction of TEMPO-oxidized cellulose nanofibrils in water. Cellulose 2016, 23, 3449-3462.

(46) Bergenstråhle, M.; Mazeau, K.; Berglund, L. A. Molecular modeling of interfaces between cellulose crystals and surrounding molecules: Effects of caprolactone surface grafting. European Polymer Journal 2008, 44, 3662-3669.

(47) Zhou, A.; Tam, L.-h.; Yu, Z.; Lau, D. Effect of moisture on the mechanical properties of CFRP-wood composite: An experimental and atomistic investigation. Composites Part B: Engineering 2015, 71, 63-73.

(48) Chen, P.; Nishiyama, Y.; Wohlert, J.; Lu, A.; Mazeau, K.; Ismail, A. E. Translational Entropy and Dispersion Energy Jointly Drive the Adsorption of Urea to Cellulose. J. Phys. Chem. B 2017, 121, 2244-2251.

(49) Fan, B.; Maranas, J. K. Coarse-grained simulation of cellulose I $\beta$ with application to long fibrils. Cellulose 2015, 22, 31-44. 
(50) Glass, D. C.; Moritsugu, K.; Cheng, X.; Smith, J. C. REACH Coarse-Grained Simulation of a Cellulose Fiber. Biomacromolecules 2012, 13, 2634-2644.

(51) López, C. A.; Bellesia, G.; Redondo, A.; Langan, P.; Chundawat, S. P. S.; Dale, B. E.; Marrink, S. J.; Gnanakaran, S. MARTINI coarse-grained model for crystalline cellulose microfibers. J Phys Chem B 2015, 119, 465-473.

(52) Wohlert, J.; Berglund, L. A. A Coarse-Grained Model for Molecular Dynamics Simulations of Native Cellulose. J. Chem. Theory Comput. 2011, 7, 753-760.

(53) López, C. A.; Rzepiela, A. J.; de Vries, A. H.; Dijkhuizen, L.; Hünenberger, P. H.; Marrink, S. J. Martini Coarse-Grained Force Field: Extension to Carbohydrates. J. Chem. Theory Comput. 2009, 5, 3195-3210.

(54) Mehandzhiyski, A. Y.; Zozoulenko, I. Computational Microscopy of PEDOT:PSS/Cellulose Composite Paper. ACS Appl. Energy Mater. 2019, 2, 3568-3577.

(55) Poma, A. B.; Chwastyk, M.; Cieplak, M. Elastic moduli of biological fibers in a coarsegrained model: crystalline cellulose and $\beta$-amyloids. Phys Chem Chem Phys 2017, 19, 28195-28206.

(56) Poma, A. B.; Chwastyk, M.; Cieplak, M. Coarse-grained model of the native cellulose I $\alpha$ and the transformation pathways to the I $\beta$ allomorph. Cellulose 2016, 23, 15731591.

(57) Mehandzhiyski, A. Y.; Rolland, N.; Garg, M.; Wohlert, J.; Linares, M.; Zozoulenko, I. A novel supra coarse-grained model for cellulose. Cellulose 2020, 27, 4221-4234.

(58) Sato, T.; Nakamura, J.; Teramoto, A.; Green, M. M. Cholesteric Pitch of Lyotropic Polymer Liquid Crystals. Macromolecules 1998, 31, 1398-1405.

(59) Wensink, H. H.; Morales-Anda, L. Chiral assembly of weakly curled hard rods: Effect of steric chirality and polarity. J. Chem. Phys. 2015, 143, 144907. 
(60) Marechal, M.; Dussi, S.; Dijkstra, M. Density functional theory and simulations of colloidal triangular prisms. J. Chem. Phys. 2017, 146, 124905.

(61) Dussi, S.; Dijkstra, M. Entropy-driven formation of chiral nematic phases by computer simulations. Nature Communications 2016, 7, 1-10.

(62) Dussi, S.; Belli, S.; van Roij, R.; Dijkstra, M. Cholesterics of colloidal helices: Predicting the macroscopic pitch from the particle shape and thermodynamic state. $J$. Chem. Phys. 2015, 142, 074905.

(63) Cinacchi, G.; Ferrarini, A.; Giacometti, A.; Kolli, H. B. Cholesteric and screw-like nematic phases in systems of helical particles. J. Chem. Phys. 2017, 147, 224903.

(64) Kolli, H. B.; Cinacchi, G.; Ferrarini, A.; Giacometti, A. Chiral self-assembly of helical particles. Faraday Discuss. 2016, 186, 171-186.

(65) Kolli, H. B.; Frezza, E.; Cinacchi, G.; Ferrarini, A.; Giacometti, A.; Hudson, T. S. Communication: From rods to helices: Evidence of a screw-like nematic phase. $J$. Chem. Phys. 2014, 140, 081101.

(66) Pelcovits, R. A. Cholesteric pitch of rigid and semi-flexible chiral liquid crystals. Liquid Crystals 1996, 21, 361-364.

(67) Odijk, T. Pitch of a polymer cholesteric. J. Phys. Chem. 1987, 91, 6060-6062.

(68) Straley, J. P. Theory of piezoelectricity in nematic liquid crystals, and of the cholesteric ordering. Phys. Rev. A 1976, 14, 1835-1841.

(69) Straley, J. P. The Gas of Long Rods as a Model for Lyotropic Liquid Crystals. Molecular Crystals and Liquid Crystals 1973, 22, 333-357.

(70) Straley, J. P. Frank Elastic Constants of the Hard-Rod Liquid Crystal. Phys. Rev. A 1973, 8, 2181-2183. 
(71) Onsager, L. The Effects of Shape on the Interaction of Colloidal Particles. Annals of the New York Academy of Sciences 1949, 51, 627-659.

(72) Růžička, v.; Wensink, H. H. Simulating the pitch sensitivity of twisted nematics of patchy rods. Soft Matter 2016, 12, 5205-5213.

(73) Wensink, H. H.; Jackson, G. Cholesteric order in systems of helical Yukawa rods. J. Phys.: Condens. Matter 2011, 23, 194107.

(74) Wu, L.; Sun, H. Manipulation of cholesteric liquid crystal phase behavior and molecular assembly by molecular chirality. Phys. Rev. E 2019, 100, 022703.

(75) Wu, L.; Sun, H. Cholesteric ordering predicted using a coarse-grained polymeric model with helical interactions. Soft Matter 2018, 14, 344-353.

(76) Li, Z.-W.; Zhu, Y.-L.; Lu, Z.-Y.; Sun, Z.-Y. A versatile model for soft patchy particles with various patch arrangements. Soft Matter 2016, 12, 741-749.

(77) Li, Z.-W.; Zhu, Y.-L.; Lu, Z.-Y.; Sun, Z.-Y. General patchy ellipsoidal particle model for the aggregation behaviors of shape- and/or surface-anisotropic building blocks. Soft Matter 2018, 14, 7625-7633.

(78) Ding, S.-Y.; Himmel, M. E. The Maize Primary Cell Wall Microfibril: A New Model Derived from Direct Visualization. J. Agric. Food Chem. 2006, 54, 597-606.

(79) Atalla, R. H.; VanderHart, D. L. The role of solid state 13C NMR spectroscopy in studies of the nature of native celluloses. Solid State Nuclear Magnetic Resonance 1999, 15, 1-19.

(80) Nishiyama, Y.; Langan, P.; Chanzy, H. Crystal Structure and Hydrogen-Bonding System in Cellulose I $\beta$ from Synchrotron X-ray and Neutron Fiber Diffraction. J. Am. Chem. Soc. 2002, 124, 9074-9082. 
(81) Habibi, Y.; Lucia, L. A.; Rojas, O. J. Cellulose Nanocrystals: Chemistry, SelfAssembly, and Applications. Chem. Rev. 2010, 110, 3479-3500.

(82) Plimpton, S. Fast Parallel Algorithms for Short-Range Molecular Dynamics. Journal of Computational Physics 1995, 117, 1-19.

(83) Chen, W.; Zhu, Y.; Cui, F.; Liu, L.; Sun, Z.; Chen, J.; Li, Y. GPU-Accelerated Molecular Dynamics Simulation to Study Liquid Crystal Phase Transition Using CoarseGrained Gay-Berne Anisotropic Potential. PLOS ONE 2016, 11, e0151704.

(84) Zhao, Z.; Shklyaev, O. E.; Nili, A.; Mohamed, M. N. A.; Kubicki, J. D.; Crespi, V. H.; Zhong, L. Cellulose Microfibril Twist, Mechanics, and Implication for Cellulose Biosynthesis. J. Phys. Chem. A 2013, 117, 2580-2589.

(85) Allen, M. P.; Germano, G. Expressions for forces and torques in molecular simulations using rigid bodies. Molecular Physics 2006, 104, 3225-3235.

(86) Vácha, R.; Frenkel, D. Relation between Molecular Shape and the Morphology of Self-Assembling Aggregates: A Simulation Study. Biophys J 2011, 101, 1432-1439.

(87) Besl, P. J.; McKay, N. D. A method for registration of 3-D shapes. IEEE Transactions on Pattern Analysis and Machine Intelligence 1992, 14, 239-256.

(88) Hub, J. S.; de Groot, B. L.; van der Spoel, D. g_wham-A Free Weighted Histogram Analysis Implementation Including Robust Error and Autocorrelation Estimates. J. Chem. Theory Comput. 2010, 6, 3713-3720.

(89) Mazeau, K.; Rivet, A. Wetting the (110) and (100) Surfaces of I $\beta$ Cellulose Studied by Molecular Dynamics. Biomacromolecules 2008, 9, 1352-1354.

(90) Dufresne, A. Nanocellulose: From Nature to High Performance Tailored Materials; Walter de Gruyter GmbH \& Co KG, 2017. 
(91) Muthoka, R. M.; Kim, H. C.; Kim, J. W.; Zhai, L.; Panicker, P. S.; Kim, J. Steered Pull Simulation to Determine Nanomechanical Properties of Cellulose Nanofiber. Materials 2020, 13, 710 .

(92) Khouri, S.; Shams, M.; Tam, K. C. Determination and prediction of physical properties of cellulose nanocrystals from dynamic light scattering measurements. J Nanopart Res 2014, 16, 2499.

(93) De Souza Lima, M. M.; Wong, J. T.; Paillet, M.; Borsali, R.; Pecora, R. Translational and Rotational Dynamics of Rodlike Cellulose Whiskers. Langmuir 2003, 19, 24-29.

(94) Mao, Y.; Liu, K.; Zhan, C.; Geng, L.; Chu, B.; Hsiao, B. S. Characterization of Nanocellulose Using Small-Angle Neutron, X-ray, and Dynamic Light Scattering Techniques. J Phys Chem B 2017, 121, 1340-1351.

(95) Boluk, Y.; Danumah, C. Analysis of cellulose nanocrystal rod lengths by dynamic light scattering and electron microscopy. JNR 2014, 16, 2174.

(96) Nyström, G.; Arcari, M.; Adamcik, J.; Usov, I.; Mezzenga, R. Nanocellulose Fragmentation Mechanisms and Inversion of Chirality from the Single Particle to the Cholesteric Phase. ACS Nano 2018, 12, 5141-5148.

(97) Zhu, Y.-L.; Pan, D.; Li, Z.-W.; Liu, H.; Qian, H.-J.; Zhao, Y.; Lu, Z.-Y.; Sun, Z.Y. Employing multi-GPU power for molecular dynamics simulation: an extension of GALAMOST. Molecular Physics 2018, 116, 1065-1077.

(98) Zhu, Y.-L.; Liu, H.; Li, Z.-W.; Qian, H.-J.; Milano, G.; Lu, Z.-Y. GALAMOST: GPU-accelerated large-scale molecular simulation toolkit. Journal of Computational Chemistry 2013, 34, 2197-2211.

(99) Beck-Candanedo, S.; Roman, M.; Gray, D. G. Effect of Reaction Conditions on 
the Properties and Behavior of Wood Cellulose Nanocrystal Suspensions. Biomacromolecules 2005, 6, 1048-1054.

(100) Honorato-Rios, C.; Bruckner, J.; Schütz, C.; Wagner, S.; Tosheva, Z.; Bergström, L.; Lagerwall, J. P. F. Liquid Crystals with Nano and Microparticles; Series in Soft Condensed Matter Volume 7; WORLD SCIENTIFIC, 2014; Vol. Volume 7; pp 871-897.

(101) Chopin, J.; Kudrolli, A. Helicoids, Wrinkles, and Loops in Twisted Ribbons. Phys. Rev. Lett. 2013, 111, 174302.

(102) Chopin, J.; Démery, V.; Davidovitch, B. Roadmap to the Morphological Instabilities of a Stretched Twisted Ribbon. J Elast 2015, 119, 137-189.

(103) Zhang, M.; Grossman, D.; Danino, D.; Sharon, E. Shape and fluctuations of frustrated self-assembled nano ribbons. Nature Communications 2019, 10, 1-7.

(104) Stukowski, A. Visualization and analysis of atomistic simulation data with OVITO-the Open Visualization Tool. Modelling Simul. Mater. Sci. Eng. 2009, 18, 015012.

(105) Wang, P.-X.; Hamad, W. Y.; MacLachlan, M. J. Structure and transformation of tactoids in cellulose nanocrystal suspensions. Nature Communications 2016, 7, 1-8.

(106) Khadem, S. A.; Rey, A. D. Theoretical Platform for Liquid-Crystalline Self-Assembly of Collagen-Based Biomaterials. Front. Phys. 2019, 7.

(107) Gobeaux, F.; Belamie, E.; Mosser, G.; Davidson, P.; Panine, P.; Giraud-Guille, M.-M. Cooperative Ordering of Collagen Triple Helices in the Dense State. Langmuir 2007, 23, 6411-6417.

(108) Khadem, S. A.; Rey, A. D. Thermodynamic modelling of acidic collagenous solutions: from free energy contributions to phase diagrams. Soft Matter 2019, 15, 1833-1846. 
Graphical TOC Entry

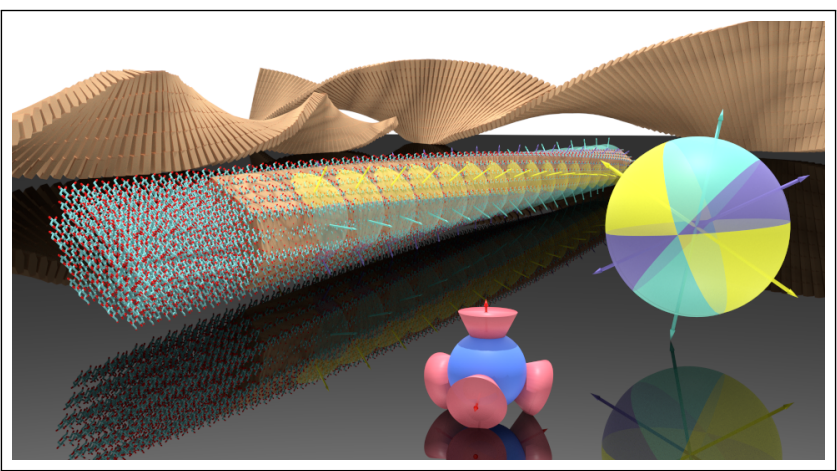

